Supporting Information for

\title{
An Approach to Extract Rate Constants from Reaction-Diffusion Dynamics in a Microchannel
}

\author{
Jean-Baptiste Salmon, ${ }^{*}$ Claire Dubrocq, and Patrick Tabeling \\ Microfluidique, Mems et Nanostructures, ESPCI, \\ 10 rue Vauquelin, 75005 Paris, FRANCE \\ Sandrine Charier, Damien Alcor, and Ludovic Jullien \\ CNRS UMR 8640 Département de Chimie, École Normale Supérieure, \\ 24 rue Lhomond, 75231 Paris cedex 5, FRANCE \\ Fabien Ferrage \\ New York Structural Biology Center, \\ 89 Convent avenue 10027 New York, NY USA
}

\begin{abstract}
\end{abstract}
1. Comparison between the solutions of the $2 \mathrm{~d}$ model and the averaged $3 \mathrm{~d}$ model

2. Measurement of the protonation constant $K_{e}$ of the coumarin

3. Nuclear Magnetic Resonance relaxation experiments

4. References

${ }^{*}$ Now at LOF, CNRS-Rhodia, 178 avenue du docteur Schweitzer, 33608 Pessac cedex, FRANCE. Fax: 0033556464790; Electronic address: jean-baptiste.salmon-exterieur@eu.rhodia.com 


\section{Comparison between the solutions of the $2 \mathrm{~d}$ model and the averaged $3 \mathrm{~d}$ model}

We study Eqs. (2) in the case of a Poiseuille velocity profile along the $Z$-direction. No appropriate nondimensionalized variables can be used to retrieve the rate constant $k$ from these equations, because the length scale $d$ is now present. If one writes:

$$
x=X /\left(\mathrm{Pe}_{a} d\right), y=Y / d, z=Z / d,
$$

where $\mathrm{Pe}_{a}=v d^{2} / D_{a}$ is the Péclet number associated to $\mathrm{A}$, and $v$ the velocity in the midplane of the channel $(Z=0)$, Eqs. (2) read

$$
\begin{aligned}
& {\left[1-4 z^{2}\right] \partial_{x} a=\operatorname{Da}(-a b+\Gamma \beta c) / \beta^{2}+\left(\partial_{y}^{2}+\partial_{z}^{2}\right) a} \\
& {\left[1-4 z^{2}\right] \partial_{x} b=\operatorname{Da}(-a b+\Gamma \beta c)+\left(\partial_{y}^{2}+\partial_{z}^{2}\right) b / \chi^{2},} \\
& {\left[1-4 z^{2}\right] \partial_{x} c=\operatorname{Da}(-a b+\Gamma \beta c) / \beta+\left(\partial_{y}^{2}+\partial_{z}^{2}\right) c / \chi^{2},}
\end{aligned}
$$

where

$$
\mathrm{Da}=\frac{k a_{0} d^{2}}{D_{a}}=\beta \frac{\tau_{d}}{\tau_{r}},
$$

is the Damköhler number which compares the diffusion time over the height of the microchannel $\tau_{d}$, and the characteristic time of the reaction $\tau_{r}$. With these nondimensionalized variables, the $2 \mathrm{~d}$ regime (where concentrations are homogeneous over $d$ ) is reached for $x \gtrsim 1$, and the local chemical equilibrium is reached for $x \gtrsim 1 /$ Da. A complete description of the various solutions and regimes of this model is left for future publication. Here we only focus on the case $\beta=\chi=\Gamma=1$ to discuss the difference between the solutions of the $2 \mathrm{~d}$ model described by Eqs. (6), and the averaged solutions of the $3 \mathrm{~d}$ model described by Eqs. (S-2). Moreover, the results derived below do not depend drastically on the specific choice $\beta=\chi=\Gamma=1$.

Figure S-1 shows the solution of Eqs. (S-2) with Da $=10^{3}$ for $x<0.03$. More precisely, Fig. S-1(a) displays the map of the concentration of $c$ averaged over the height of the microchannel, i.e. $\bar{c}(x, y)=\int \mathrm{d} z c(x, y, z)$. Figures $\mathrm{S}-1(\mathrm{~b})-(\mathrm{d})$ show slices in the plane $(y-z)$ of the concentration field at three distances downstream. As anticipated from the dispersion of the residence times across the channel height due to the Poiseuille velocity profile, the width of the diffusion zone is larger at top and bottom of the channel than in the midplane [1]. In the case where $\chi \neq 1$ (difference of diffusion coefficients) or $\beta \neq 1$ (difference of 

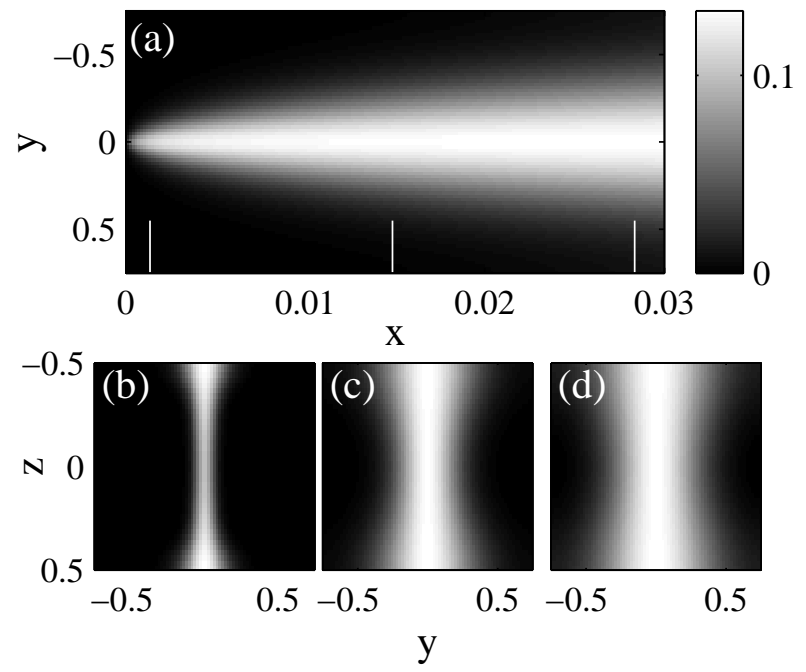

FIG. S-1: (a) Averaged concentration map $\bar{c}(x, y)$ computed using Eqs. (S-2) in the case $\beta=\chi=$ $\Gamma=1$ and $\mathrm{Da}=10^{3}$. (b)-(d) Slices of the concentration field in the plane $y$ - $z$ at the distances $x=1.3510^{-3}, 1.4810^{-2}$, and $2.8410^{-2}$ indicated by white ticks on (a).

the initial concentrations), asymmetric concentration fields as observed experimentally in Ref. [1] can be reproduced by Eqs. (S-2).

Figure S-2(a) displays the difference between the solution of Eqs. (6) and the solution of Eqs. (S-2) averaged over the height of the microchannel, in the case $\mathrm{Da}=10^{3}$ that is close to our present experimental conditions. This map is plotted against $x$ and $y$ defined by Eqs. (S-1). Let us recall that in these units, the $2 \mathrm{~d}$ regime is reached for $x \gtrsim 1$ and the diffusive regime for $x \gtrsim 1 / \mathrm{Da} \approx 10^{-3}$. Several remarks may be done. First, the differences between the $2 \mathrm{~d}$ model and the averaged solution of the $3 \mathrm{~d}$ model are small since the maximal difference is of the order of $10^{-2}$ (the concentration at equilibrium is about 0.13 ). Secondly, the differences between the $2 \mathrm{~d}$ solution and the averaged $3 \mathrm{~d}$ solution decrease as $x$ increases. This is due to the molecular diffusion along the $z$-direction, which homogeneizes the concentrations over the height of the channel. For $x \gtrsim 1$, we checked that the difference was almost null at each point downstream. Finally, the maximal differences between the two solutions occur along the edge of the diffusion cone of the product of the reaction. The difference is thus almost null in the region of the maximum of production of $c$ (the $y=0$ line, for $\chi=\beta=1$ ). Figure S-2(b) displays the maximal values of the concentration along the $y$-direction vs. $x$, in the case of the $2 \mathrm{~d}$ model and the averaged $3 \mathrm{~d}$ model, i.e. $c_{\max }(x)$ 


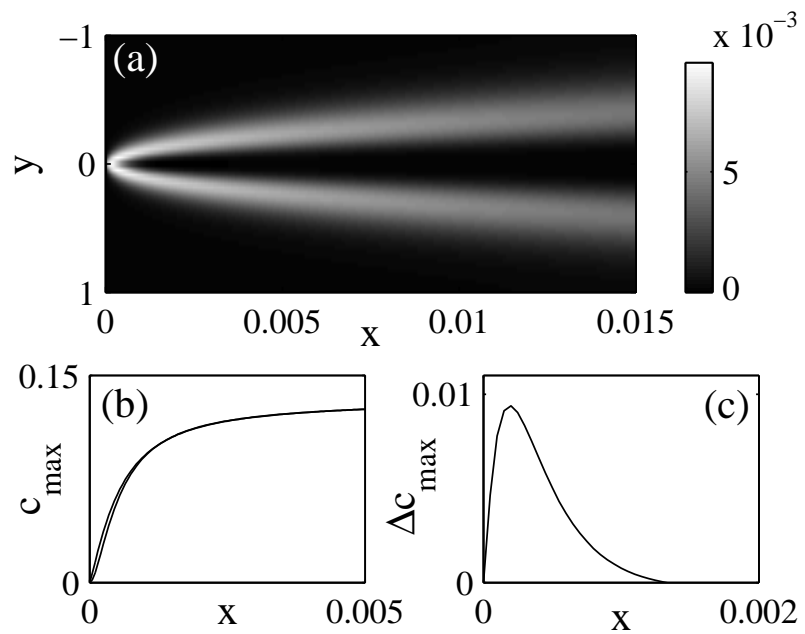

FIG. S-2: (a) Difference of the concentration map of $c$ computed using Eqs. (6), and the concentration map of $c$ averaged over the height of the microchannel computed using Eqs. (S-2). (b) Maximum of the concentration at each position $y$ vs. $x$ for the $2 \mathrm{~d}$ and the $3 \mathrm{~d}$ model. The two curves are almost indistiguishible. (c) Difference between the two curves displayed in (b).

and $\bar{c}_{\text {max }}(x)$. As shown in Figure S-2(c), the maximal difference is small $\left(\Delta c_{\max } \approx 0.01\right)$ and occurs at about $x \approx 210^{-4}$ : the theoretical solutions $c_{\max }(x)$ of the $2 \mathrm{~d}$ model can be used to extract the rate constant from experimental data if the measured concentrations are averaged over the height of the microchannel.

To get more quantitative results, we simulated Eqs. (S-2) for several Da. Figure S-3 displays $\Delta c_{\max }$, the maximal difference between the solutions of the $2 \mathrm{~d}$ and the averaged 3d model along the line $y=0$, vs. Da. Figure S-3 reveals three regimes. (i) If Da $<1$, the error is almost null. In this case, the reaction is slow compared to the diffusion time across the height of the microchannel, and the kinetics regime occurs when the concentration fields are homogeneous along $z$. (ii) If $1<\mathrm{Da}<10^{4}$, the difference between the $2 \mathrm{~d}$ and $3 \mathrm{~d}$ solutions increases with increasing Da, but remains small. For such Da values, the kinetic regime occurs when the concentration fields are not uniform along the $z$-direction. (iii) When $\mathrm{Da}>10^{4}$, the maximal difference seems to saturate at a value of about $0.06 c_{e q}$. For such values of the Damköhler number, the reaction is almost instantaneous, and the concentration fields quickly evolves according diffusive processes. 


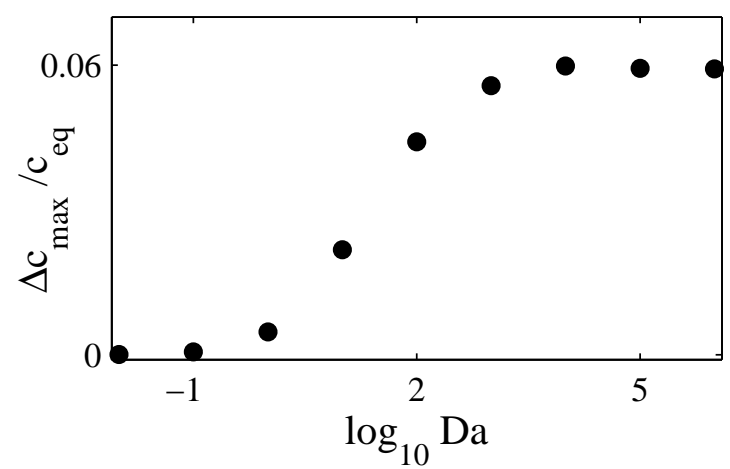

FIG. S-3: Normalized maximal difference between the $2 \mathrm{~d}$ model and the averaged $3 \mathrm{~d}$ model vs. $\mathrm{Da}$, in the case $\chi=\beta=\Gamma=1$. The values are normalized with the concentration at the chemical equilibrium $c_{e q} \approx 0.13$.

Measurement of the protonation constant $K_{e}$ of the coumarin 

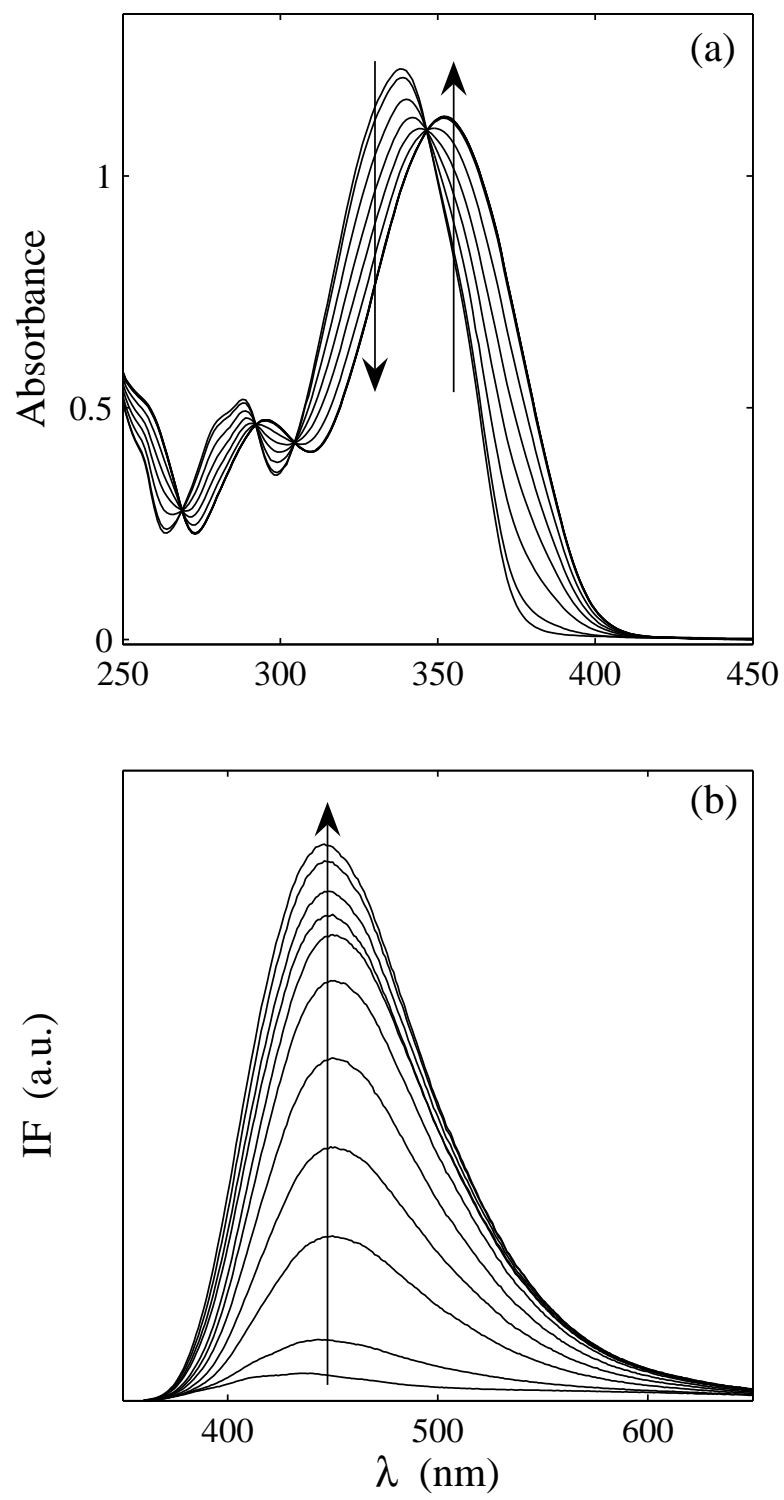

FIG. S-4: Evolution of the absorption (a) and emission (b) spectra of the coumarin in acetonitrile (total concentration $[\text { coumarin }]_{\text {tot }}=1.110^{-4} \mathrm{M}$ ) upon increasing the proton concentration. (a) UV-Vis absorbance; (b) Emission intensity IF for $\lambda_{\mathrm{exc}}=348 \mathrm{~nm} . R=\left[\mathrm{H}^{+}\right]_{\text {tot }} /[\text { coumarin }]_{\text {tot }}=0$, $0.21,0.42,0.63,0.83,1.04,1.25,1.46,1.88,2.30,2.71,3.13$. The arrows precise the direction of the evolution of the curves upon increasing $R$.

\section{Nuclear Magnetic Resonance relaxation experiments}



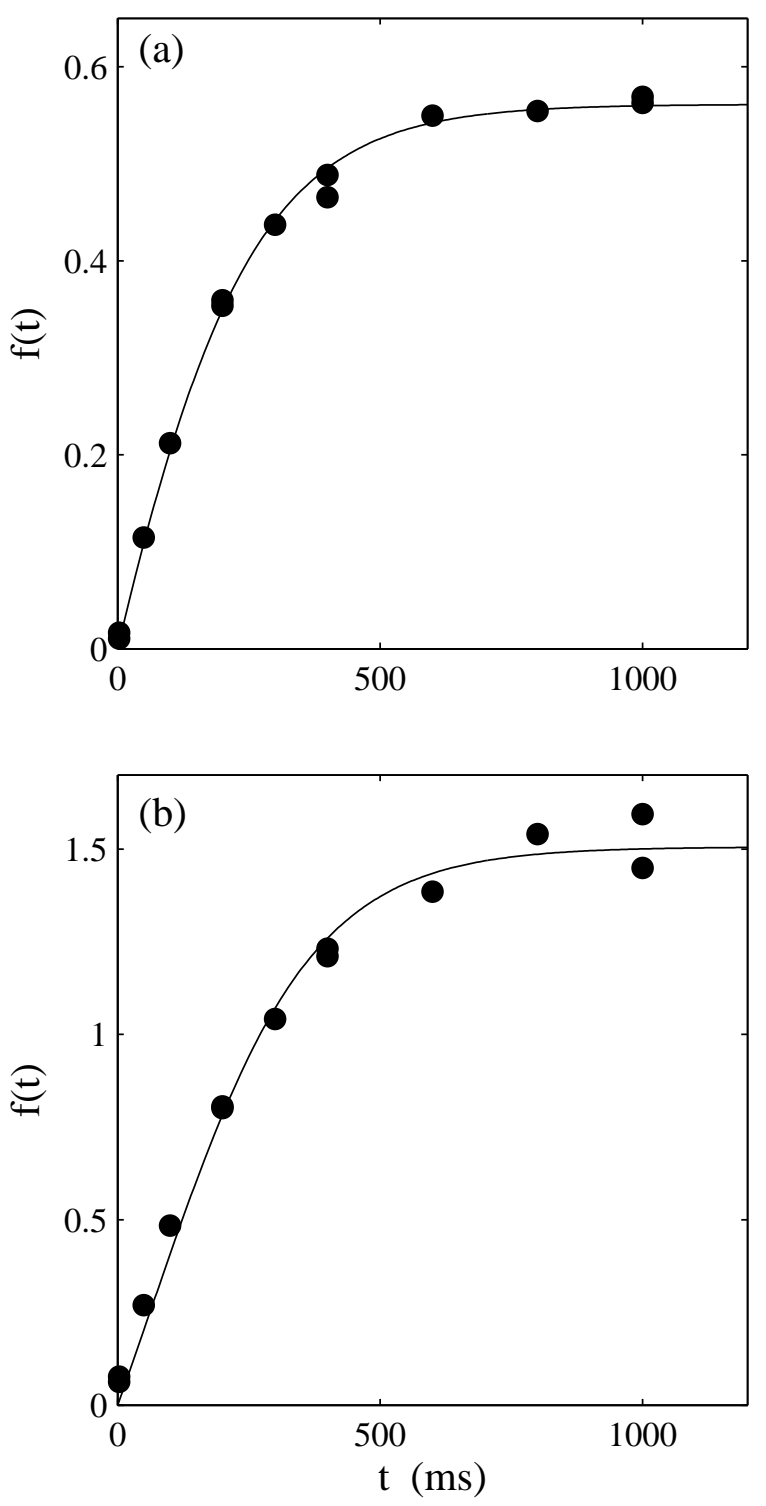

FIG. S-5: Analysis of the results from NMR experiments. (a) Initial selection of the basic form, and (b) of the acidic form. The continuous lines are the best fits of the experimental data according to Eq. (10).

[1] R. F. Ismagilov, A. D. Stroock, P. J. A. Kenis, G. Whitesides, and H. A. Stone, Appl. Phys. Lett., 2000, 76, 2376. 